\title{
Jump Events in a 3D Edwards-Anderson Spin Glass
}

\author{
Daniel A. Mártin* and José Luis Iguain ${ }^{\dagger}$ \\ Instituto de Física de Mar del Plata (IFIMAR), \\ CONICET and Facultad de Ciencias Exactas y Naturales, \\ Universidad Nacional de Mar del Plata, Funes 3350, Mar del Plata, Argentina
}

(Dated: December 28, 2016)

\begin{abstract}
The statistical properties of infrequent particle displacements, greater than a certain distance, is known as jump dynamics in the context of structural glass formers. We generalize the concept of jump to the case of a spin glass, by dividing the system in small boxes, and considering infrequent cooperative spin flips in each box. We perform numerical simulations for the Edwards-Anderson 3D model, and study how the properties of these jumps depend on the waiting time after a quench. Similar to the results for structural glasses, we find that while jump frequency depends strongly on time, jump duration and jump length are roughly stationary. At odds with some results reported on studies of structural glass formers, at long enough times, the rest time between jumps varies as the inverse of jump frequency. We give a possible explanation for this discrepancy.

PACS numbers: $\quad$ 75.10.Nr, 64.70.P-,05.50.+q
\end{abstract}

\section{INTRODUCTION}

Glass formers and spin glasses have been the subject of intense theoretical, experimental and numerical research in the last decades (see for instance, [1-3] and references therein). These systems present similar phenomenology (see for instance [4]), analogous techniques have been developed in both $[5,6]$, and their relationship has been proved [7-9]. Thus, in spite of the details about composition, structure or kind of degrees of freedom, it is common to use the word glassy to refer to any material with a dynamics qualitatively similar to that of a glass [10].

The study of glassy systems poses a series of issues, related to the impossibility to reach equilibrium below the glass transition temperature $T_{g}$. In this case, the system evolves for ever. However, the time dependence of the one-time observables, like density, energy, pressure or magnetization (in a magnetic system) is quite weak and hard to appreciate. Aging effects are more clearly observed in the behavior of two-time observables, like the autocorrelation $C\left(t_{2}, t_{1}\right)$ and response $R\left(t_{2}, t_{1}\right)$ functions, which do not depend only on the time difference $t_{2}-t_{1}$ but on $t_{1}$ and $t_{2}$ (see, for example, [11-13])

An alternative is to study aging-to-equilibrium [14, 15], i. e., the out-of-equilibrium relaxation at a temperature above $T_{g}$. When a system is quenched from an equilibrium state at a temperature $T_{0}$ to a lower temperature $T_{F}>T_{g}$, the transition to equilibrium takes place on a time scale related to the equilibrium relaxation time $\tau\left(T_{F}\right)$, which diverges at $T_{g}$. It is expected that some aspects of glassy dynamics will be revealed for $t \ll \tau\left(T_{F}\right)$. In [15], two-time correlation functions were studied in both aging-to-equilibrium and aging regimes for a simple glass former. In [14], the authors analyzed the aging-toequilibrium dynamics for the strong glass former $\mathrm{SiO}_{2}$.

\footnotetext{
* danielalejandromartin@gmail.com

† iguain@mdp.edu.ar
}

Most of the studies about structural glass formers systems focus on macroscopic or global observables. Nevertheless, in recent years microscopic (i. e., involving a single or few particle) actions have been analyzed [16-20] in structural glass formers, with the goal of understanding better the mechanisms responsible for aging. In this context, aging in spin glasses was also analyzed via the probability density function of the heat transfer over a short time between system and heat bath [21]. In [16], the microscopic dynamics of $\mathrm{SiO}_{2}$ were analyzed and related to the macroscopic dynamics. After a quench, jump events, i. e., particle movements greater than a certain threshold, were detected and their statistical properties were analyzed. The authors found that the number of jumping particles decreases strongly with $t$, the time elapsed since the quench, reaching equilibrium at times compatible with $\tau\left(T_{f}\right)$. Other properties of jumps, as average length, time duration, and surprisingly rest time between consecutive jumps do not depend on $t$. In contrast, in [22] the authors studied the distribution functions of the firstpassage time, and the persistence time, and found that both quantities evolve with time.

Jump events are not only closely related to macroscopic evolution observables like diffusivity [23] but constitute an important ingredient in the relaxation of glass formers. They provide a link among structure and dynamics, by giving a more quantitative idea of the cage effect. This is the case in [24], where, for a 2D model at low temperature, it is found that non jumping particles were more likely in highly ordered environments. The relationship among jumps and dynamical heterogeneities has also been established [25].

In this work, we study some microscopic aspects of the aging-to-equilibrium and aging dynamics of the well known 3D Edwards-Anderson (EA) spin-glass model [2628]. This model has been extensively studied in a macroscopic way, but also microscopic results have been reported $[29,30]$, including the existence of a backbone [31]. 
A great number of lengths and correlators can be measured nowadays in spin-glasses. The analysis of these results have been useful for the development of new numerical tools, like out-of-equilibrium finite-size analysis [32], which allow to overcome the slow dynamics. The use of special techniques to avoid the effects of boundary conditions have also been employed [33]. Special strategies that take profit of conventional processors [34] or use specific hardware $[35,36]$ have been reported. Using them, simulation times closer to experimental times where reached [37].

Here we are not interested in understanding the longtime low-temperature regime of a large-size system. We use this model as an example to study the dynamics of jumps in a spin glass. We start by generalizing the concept of jump, introduced originally for structural glass formers, to the case of a spin glass. We wish to analyze whether the statistical properties of jump events reported in [16] can be reproduced in a glassy system composed of spins, instead of moving particles.

For the EA model, we find that microscopic and macroscopic relaxation times behave similarly. We also find that some variables depend strongly on time while others are nearly stationary. Thus, most of the results in [16] for a structural glass former hold for this spin glass model. For instance, jump length and jump duration are roughly stationary, while the number of jumps depends strongly on $t$. On the other hand, we find that rest time between jumps, which is independent of time in [16], has an inverse relation to jump frequency for the EA model. We draw a plausible explanation for this discrepancy.

The paper is organized as follows. In Sec. II, we describe the model and observables. In Sec. III, we define an equilibration time based on macroscopic observables. Main results, i.e., evolution of microscopic observables, are presented in Sec. IV. In Sec. V, we discuss our findings and future directions, and draw our conclusions. In Appendix we explain the procedure to estimate the equilibration time, and show the consistency with the equilibration time for energy.

\section{MODEL AND OBSERVABLES}

We study the Gaussian EA model in a cube with $L$ spins by side, under periodic boundary conditions, defined by the Hamiltonian:

$$
H=-\sum_{\langle i, j\rangle} J_{i j} S_{i} S_{j}
$$

where the indexes $i, j$ run from 1 to $L^{3}$. Spin variables take values $S_{i}= \pm 1$ and the pairs $\langle i, j\rangle$ identify nearestneighbors. The couplings are taken randomly with a Gaussian distribution with zero mean and unit variance.

Dynamics is simulated with Metropolis algorithm. That is, at each Monte Carlo (MC) step, $N=L^{3}$ spin flip trials are performed. They are accepted or rejected according to their Boltzmann weights.
For this system, $T_{g} \simeq 0.95$ [38]. So, in order to study aging-to-equilibrium, and also aging-to-non-equilibrium, we equilibrate the system at temperature $T_{0}=3$ (we have also performed runs for $T_{0}=\infty$ ) for $10^{5} \mathrm{MC}$ steps. We define $t=0$ as the time at which the system is quenched to the final temperature $T_{f}=1.5,1.2,1.0$ and also 0.9 which is below $T_{g}$. We have run 1000 samples for each temperature, for $10^{6} \mathrm{MC}$ steps, after the quench. We have also run at least 60 samples for $10^{8} \mathrm{MC}$ steps. We will focus on the range $10^{3}<t<10^{6}$ where most interesting phenomena takes place.

In next paragraphs, we define several microscopic observables, by dividing the full system into cubic boxes of side $l_{b}$, which contain $N_{b}=l_{b}^{3}$ spins each. Unless explicitly stated, we have worked with $L=16$ and $l_{b}=4$. Then, each box $C_{i}$ is labeled with index $i$ running from 1 to 64 . We record the configuration every $\delta t=5 \mathrm{MC}$ steps which we will call a unit time.

We define the overlap between consecutive records of box $i$ as

$$
O_{i}(t)=\frac{1}{N_{b}} \sum_{j \in C_{i}} S_{j}(t) S_{j}(t-\delta t),
$$

which we compute for $t=n \delta t$, with $n$ a positive integer.

We also compute the magnetization $M_{i}$ and the energy $E_{i}$ of every box.

$$
\begin{aligned}
M_{i}(t) & =\sum_{j \in C_{i}} S_{j}(t) \\
E_{i}(t) & =\frac{1}{2} \sum_{j \in C_{i}, k} J_{j k} S_{j}(t) S_{k}(t),
\end{aligned}
$$

where $k$ is a nearest neighbor of $j$.

When the simulation ends, we calculate $\left\langle O_{i}\right\rangle$ and $\left\langle O_{i}^{2}\right\rangle$, for all boxes averaged in the time window $5.10^{5} \mathrm{MC}<t<$ $10^{6} \mathrm{MC}$. With this, we calculate $\sigma_{i}=\sqrt{\left\langle O_{i}^{2}\right\rangle-\left\langle O_{i}\right\rangle^{2}}$ In Fig. 1 we show the values of $O_{i}$ and $\sigma_{i}$ averaged over small time windows of $10000 \mathrm{MC}$ steps, for a single box $(i=1)$. We see that both quantities are nearly constant even for the lowest temperature $T=0.9<T_{g}$. This makes them reasonably robust parameters to study jumps.

Greater changes in the configuration of a single box are related to smaller values of the overlap between two consecutive configurations. We will say that box $i$ is jumping at time $t$ if

$$
O_{i}(t)<\left\langle O_{i}\right\rangle-\gamma \sigma_{i}
$$

We have taken $\gamma=3$ in most of the cases, although we have also studied $\gamma=5$. The jump starts at $t_{i}$, where $t_{i}$ is the greatest value that verifies both $t_{i}<t$ and that Eq. (4) does not hold for $t_{i}$ (i.e. $O_{i}\left(t_{i}\right) \geq\left\langle O_{i}\right\rangle-\gamma \sigma_{i}$ ). Similarly, the jump ends at $t_{f}>t$, if $t_{f}+\delta t$ is the smallest value that does not verify Eq. (4). 


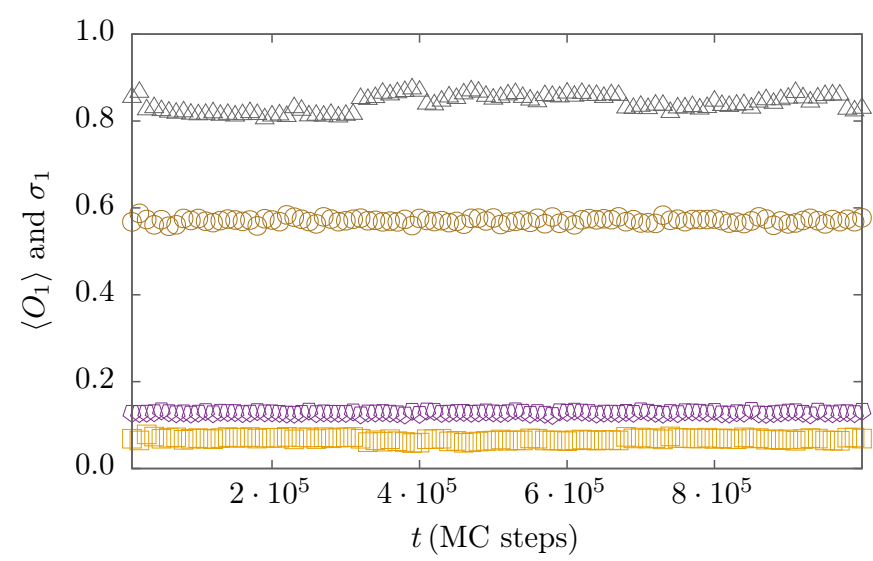

FIG. 1. (Color online) $\sigma_{1}$ and $\left\langle O_{1}\right\rangle$ of a single box, as a function of $t$, for different temperatures. Black triangles: $\left\langle O_{1}\right\rangle$ for $T_{F}=0.9$. Grey circles: $\left\langle O_{1}\right\rangle$ for $T_{F}=1.5$. Orange squares: $\sigma_{1}$ for $T_{F}=0.9$. Violet pentagons: $\sigma_{1}$ for $T_{F}=1.5$.

We define the jump frequency $\nu$, as the number of jumps per box per unit time. We measure it as a function of $t$. For each jump we define a jump duration as $d=t_{f}-t_{i}$. We define the rest time $r$, as the time the particle waits until next jump. That is, the difference between $t_{i}$ for the next jump and $t_{f}$ for the current jump. We define the jump size, $l$, using overlap values: we calculate the sum of the overlap changes over the times belonging to the jump, i.e. $l=\sum_{t_{i}<t \leq t_{f}} N_{b}-O_{i}(t)$.

We also compute some macroscopic one-time quantities as the total energy, magnetization, and number of spins that flip, and the global two-time correlation $C(t+\Delta t, t)$ :

$$
C(t+\Delta t, t)=\frac{1}{N} \sum_{i=1}^{N} S_{i}(t) S_{i}(t+\Delta t) .
$$

\section{RESULTS: MACROSCOPIC QUANTITIES}

We want to measure the time it takes to equilibrate the system, using a macroscopic variable.

Similar to [39], we find that the energy per spin, $e(t, T)$ is well fitted by $e_{\infty}+A(T) t^{-b(T)}(e(t)$ and this fit are shown in Appendix), from which a relaxation time is hard to find, so we decide to use two time correlation functions.

It would be desirable to find $\tau_{e}^{\text {ideal }}$ such that for $t>$ $\tau_{e}^{\text {ideal }}, C(t+\Delta t, t)$ does not depend on $t$. We cannot do that due to limited precision in our data. We define an auxiliary variable $\tau_{a}(t)$ such that $C\left(t+\tau_{a}(t), t\right)=K$. In our case, we choose $K=0.2$. Notice that an analogous procedure was also employed in $[14,16]$.

In Fig. 2-top, we show the two-time correlation as a function of $t$ for various $t$ for temperature $T=1.2$. In Fig. 2-bottom, we plot $\tau_{a}(t)$ for $T=1.2$. For $T_{F}>T_{g}$, the relaxation time is then $\tau_{a}^{E Q}\left(T_{F}\right)=\lim _{t \rightarrow \infty} \tau_{a}(t)$.
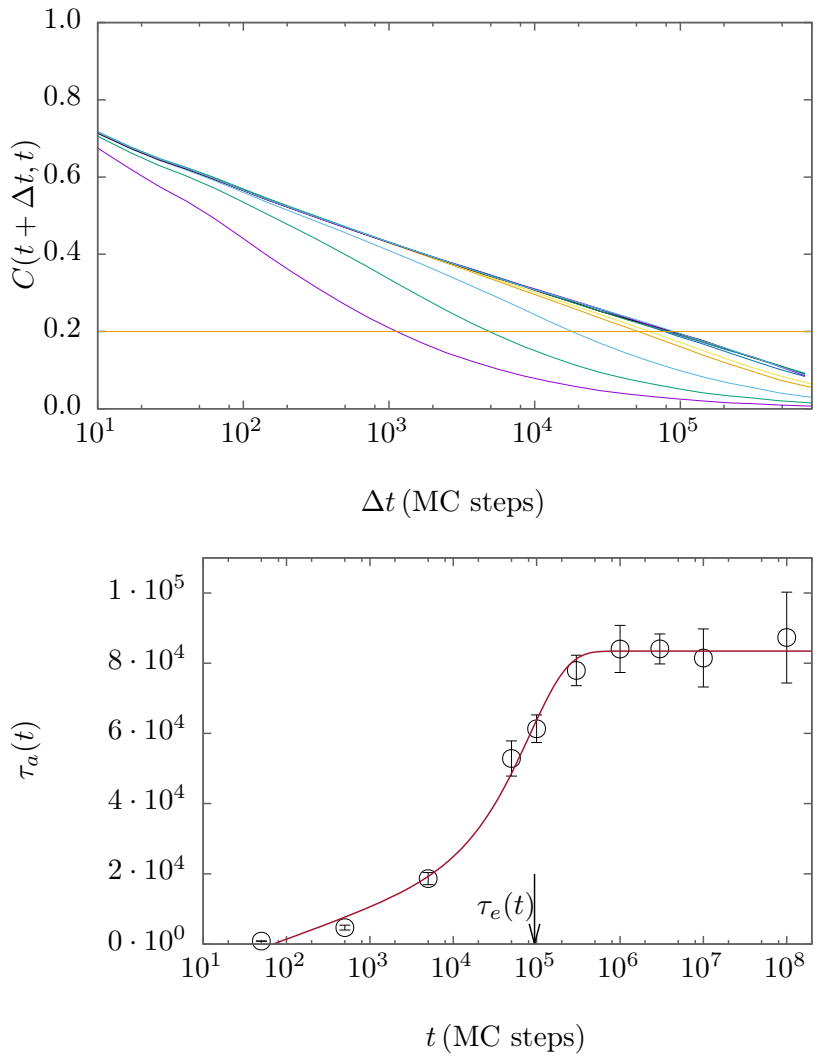

FIG. 2. (Color online) Top: Global two-time correlations for $T_{F}=1.5$, and several waiting times. From left to right, $t=5 ., 5.10,5.10^{2}, 5.10^{3}, 5.10^{4}, 10^{5}, 3.10^{5}, 10^{6}, 3.10^{6}, 10^{7}, 10^{8}$

MC steps. The horizontal line corresponds to $C(t+\Delta t, t)=0.2$. Bottom: $\tau_{a}$ as a function of $t$ for $T_{F}=1.2$ (black circles). The (red) line corresponds to the fitting function $\left(C_{1}+C_{2} \log (t)\right) e^{-t / C_{3}}+C_{4}\left(1-e^{-t / C_{3}}\right)$. The arrow indicates $\tau_{e}=C_{3}$.

Specific details on how we measure $\tau_{a}(t)$ are show in Appendix. The main point in this section is to get an estimate of macroscopic relaxation time.

From Fig. 2-bottom, we see that $\tau_{a}(t)$ grows with $t$ until it gets a constant value. To describe the growth of $\tau_{a}(t)$ at short time, and the final constant value, we have fitted $\tau_{a}(t)$ with $F(t)=\left(C_{1}+C_{2} \log (t)\right) e^{-t / C_{3}}+C_{4}\left(1-e^{-t / C_{3}}\right)$.

From this fit, we can define $\tau_{e}$, the time at which $\tau_{a}(t)$ changes from growing to constant as $\tau_{e}=C_{3}$. Using this procedure, we were able to get $\tau_{e}$ for $T=1.5,1.35$ and $T=1.2$. Although $T=1.0>T_{g}$ we were unable to estimate $\tau_{e}$ for this temperature, since we don't have enough data for long $t_{w}$.

We have checked, for $T_{F} \geq 1.2$, that $\tau_{e}$ is a good estimate of the macroscopic equilibration time for the onetime macroscopic variables, like total energy and number of flips. 


\section{RESULTS: MICROSCOPIC QUANTITIES}

We analyze the time evolution of the statistical properties of jump events after a quench.

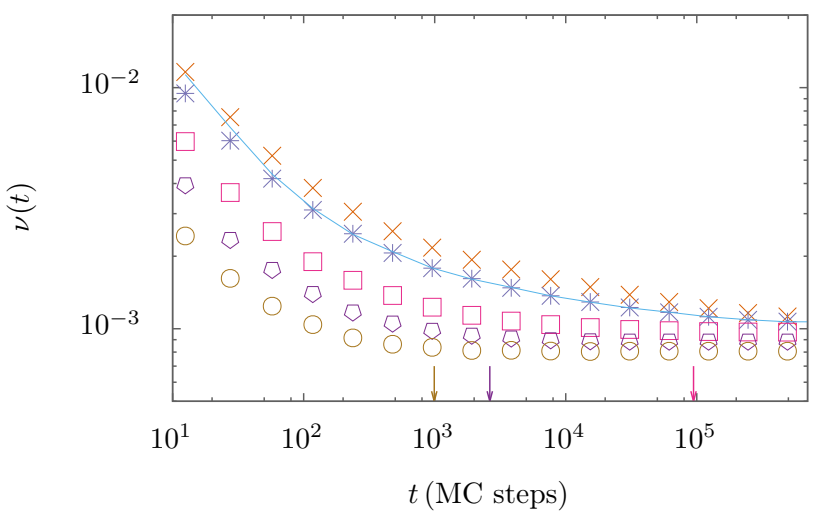

FIG. 3. (Color online) Jump frequency as a function of time. Symbols correspond to $T_{0}=3$, from top to bottom, $T_{F}=0.9$, $1.0,1.2,1.35$ and 1.5. Line with no symbols is for $T_{0}=\infty$, $T_{F}=1.0$. Arrows showing $\tau_{e}$ for $T_{F}=1.5,1.35$, and 1.2 (from left to right) have been added at figure bottom. Errors are smaller than symbol size.

In Fig. 3, we show the jump frequency as a function of time in logarithmic scale. We have added data for $T_{0}=\infty$ and $T_{F}=1.0$, which looks qualitatively similar, at least with our time windows, as data for $T_{0}=3$. This result holds for other values of $T_{F}$ (not shown). We see a steep decrease, consistent with the results of [16]. The slope is greater for lower temperatures and time for which the decreasing of $\nu$ becomes negligible are compatible with macroscopic relaxation times for temperatures $T_{F} \geq 1.2$, where we are able to measure $\tau_{e}$. We have indicated these values with arrows at the bottom of Fig. 3, to facilitate comparison.

In Fig. 4, we plot both jump duration and rest time against time. It is apparent that the first is of the order of $\delta t$, which means that most of the jump last one unit time. This is similar to what reported in [16], where average jump duration is close to time step. Jump duration decreases very slowly with temperature and has no appreciable time dependence even before $\tau_{e}$. However, at odds with [16], rest time seems to evolve with $t$. This will be further discussed in next subsection.

In Fig. 5, we show the average value jump size. Note that this quantity stabilizes before equilibration time. Even for $T=0.9$, which is below $T_{g}$, it does for $t \sim 10^{4}$.

For the sake of completeness, in Fig. 6 we show the asymptotic values of $\nu, r, d$ and $l$ for all the temperatures at which we can equilibrate the system. Jump length grows with temperature and jump duration decreases, as might be expected. Jump frequency decreases with temperature and rest time grows, which may seem odd, but it can be readily understood if we notice, as shown in Fig. 1 that $\langle O\rangle$ decreases and $\sigma$ grows with temperature. Then,

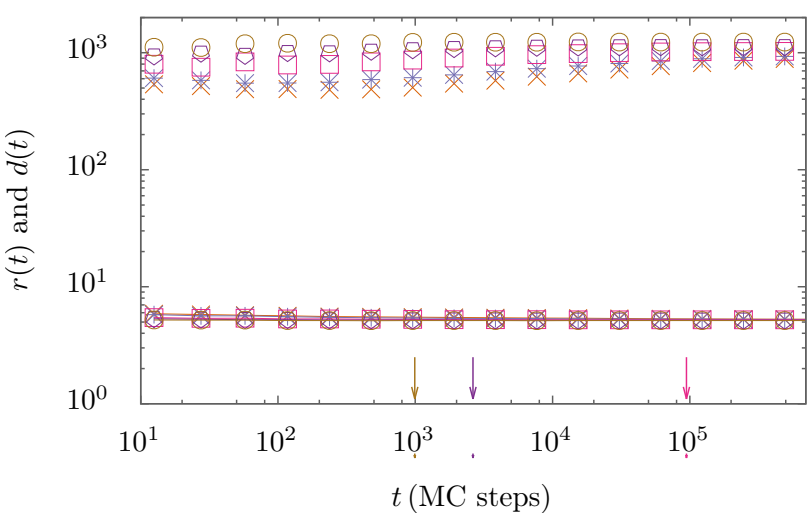

FIG. 4. (Color online) Rest time (symbols) and jump duration (lines with symbols) as a function of time. For $t>10^{5}$ there is an artificial decrease of rest time, related to the finite simulation time. Symbols and colors are the same as in Fig. 3. Results for $T_{0}=\infty$ were avoided for the sake of clarity. Errors are smaller than symbol size.

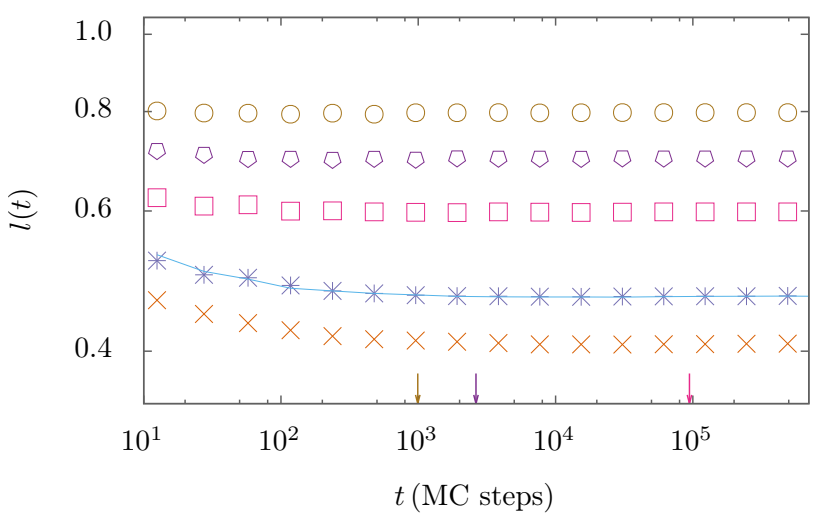

FIG. 5. (Color online) Jump size as a function of time for several temperatures. Symbols and colors are the same as in Fig. 3. Errors are smaller than symbol size.

a jump at higher temperature implies the movement of a grater amount of spins.

\begin{tabular}{|c|c|c|c|}
\hline \multirow{2}{*}{} & \multicolumn{3}{|c|}{$T_{F}$} \\
\cline { 2 - 4 } & 0.9 & 1.0 & 1.2 \\
\hline$s_{\nu}$ & $-0.082 \pm 0.005$ & $-0.054 \pm 0.004$ & $-0.013 \pm 0.002$ \\
\hline$s_{r}$ & $0.080 \pm 0.005$ & $0.053 \pm 0.004$ & $0.013 \pm 0.002$ \\
\hline
\end{tabular}

TABLE I. Long-time effective exponents for jump frequency and rest time for $\gamma=3$ (for higher temperature, the average exponents are smaller than their errors). In all cases, data were fitted in the range $10^{4}<t<5.10^{5}$. 

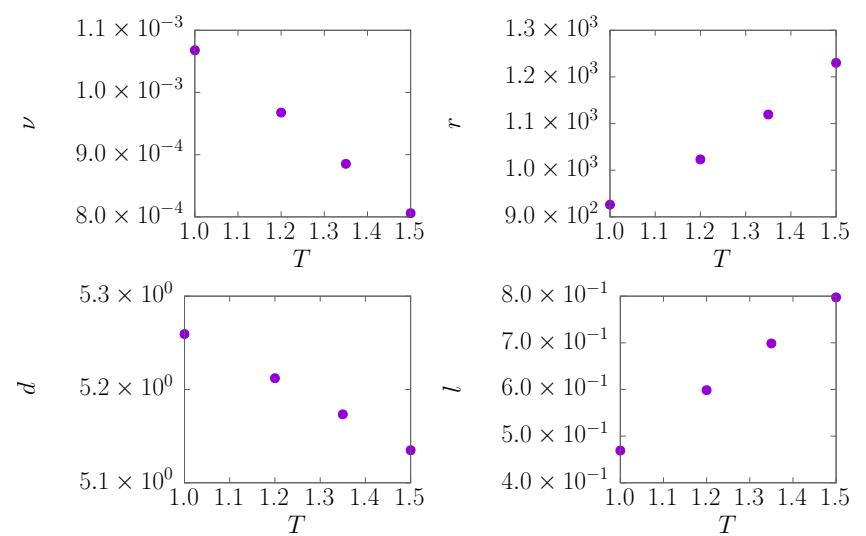

FIG. 6. Asymptotic values of $\nu, r, d$ and $l$ for $T=1, T=1.2$, $T=1.35$ and $T=1.5$. Data was taken from results in the range $10^{5}<t<10^{6}$ for all temperatures but $T=1.0$, where data was calculated in the $10^{7}<t<10^{8}$ range. Error bars are smaller than symbol size.

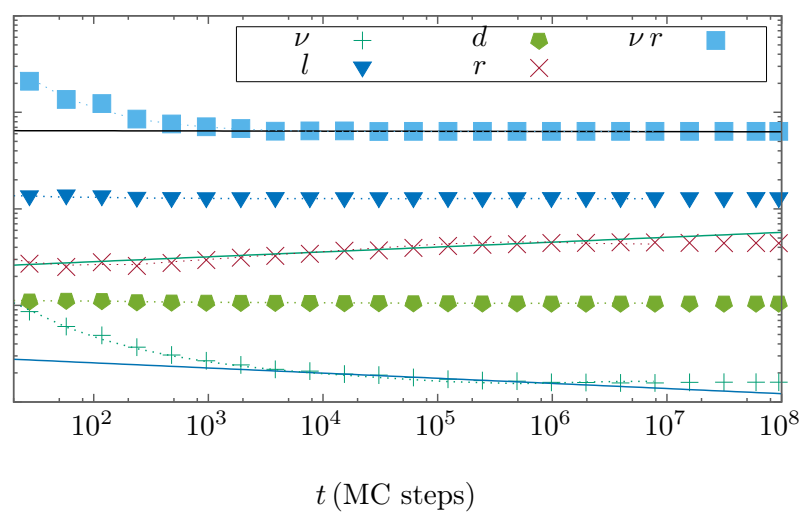

FIG. 7. Symbols show results for all observables at $T=1.0$, shifted so that its comparison becomes more clear. Errors are smaller than symbol size. Dotted lines show the same results for a bigger system: $L=32, l_{b}=4$. Straight lines are the fit of $\nu, r$, and $r \nu$, as a function of $t$, using power-law forms in the range $10^{4}<t<5.10^{5}$ (for $L=16$ data). Fitted slopes for $\nu$ and $r$ are shown in table I.

\section{A. Slopes}

To summarize, at all temperatures, some variables change in a much more pronounced way than others. This can be better appreciated in Fig. 7, where we show all the previous data for $T=1.0$ in logarithmic scale. Results are shifted (multiplied by a constant) to facilitate comparison. Let us remark that while the number of jumps decreases by about one order of magnitude, other variables have negligible changes (as in [16]), with the only exception of rest time, which grows at long times.

\begin{tabular}{|l|l|l|}
\hline \multirow{2}{*}{} & \multicolumn{2}{|l|}{ Time range } \\
\cline { 2 - 3 } & $10^{4}-510^{5}$ & $10^{5}-10^{6}$ \\
\hline$s_{\nu}$ & $-0.097 \pm 0.011$ & $-0.081 \pm 0.007$ \\
\hline$s_{r}$ & $0.047 \pm 0.014$ & $0.082 \pm 0.014$ \\
\hline
\end{tabular}

TABLE II. Effective slopes for jump frequency and rest time for $T=1, \gamma=5$ and different time windows

\section{B. Issues with rest time}

In Ref. [16], the authors study jumping particles, while in this work we study jumps of boxes. These quantities behave similarly, however we have found a discrepancy in the behavior of the rest time; which is roughly constant in [16]. Since, according to our simulations (see, for example, Fig. 4), there exist time dependence for this quantity, some words are in order.

For short enough times, i. e., for $t \ll\langle r\rangle$, most of boxes jump once or never. The results corresponding to $r(t)$ in Fig. 4 indicate that in this time interval $\left(\langle t\rangle \simeq 10^{3} \mathrm{MC}\right.$ steps) jump duration is nearly constant. For longer times $(t \gg\langle r\rangle)$, when most of jumping boxes do many jumps, it should exist some correlation between rest time and jump frequency. Since jump duration is much smaller than time between jumps, the average number of jumps per unit time, multiplied by the rest time, should be equal to the total time multiplied the number of boxes, i. e., $r \nu \simeq 1$. Thus, for long enough times, we expect that $r$ increases as $\nu$ decreases. Notice that this is what happens if Fig. 7, where $\nu, r, d, l$ but also $\nu r$ have been plotted as a function of $t$ for $T=1.0$. After a time of the order of few $\langle r\rangle, \nu r$ becomes constant

In order to get more quantitative, we try to estimate the slope of those curves. So we fit data with a function $a t^{s}$, for some constants $a$ and $s$ at $t$ about $10^{5}\left(10^{4}<\right.$ $\left.t<5.10^{5}\right)$. Notice that this function is an approximation performed in a limited range of $t$ values and breaks at larger times (at long enough times, systems above $T_{g}$ equilibrate and all slopes become 0). Functions were fitted using nonlinear least-squares Marguerite-Levenberg algorithm. The long-time $\left(10^{4}<t<510^{5}\right)$ effective exponents for jump frequency $\left(\nu(t) \sim t^{s_{\nu}}\right)$ and rest time $\left(r(t) \sim t^{s_{r}}\right)$ are opposite $\left(s_{\nu} \simeq-s_{r}\right)$ within statistical error. The same happens for other temperatures, see table I.

\section{Sensitivity of results}

In this subsection we present the results of several tests we carried out to analyze the robustness of the statistical properties of jump events.

We have checked that the outcomes for $T_{0}=3$ are similar to those for $T_{0}=\infty$. Thus, most interesting results do not depend much on $T_{0}$. Also, we found qualitatively the same results for different unit times $\delta t=2,5$ and 100 , though choosing smaller $\delta t$ makes the decreasing of 
$\nu$ more pronounced.

We have verified that doubling system size does not change the results within statistical error: see Fig. 7. We have measured the coherence length $\xi(t)$ as defined in [30]. The ratio of this length to system size is an important parameter in our study, since a change of regime, governed by finite size effects, is expected for $\xi(t) \simeq L$. In Fig. 8, we show $\xi(t)$ for $T=1.0, T=1.2$ and $T=1.5$. It is clear that, setting $L=16$, we are far from that regime. Notice also that while $\xi$ is a slightly growing function

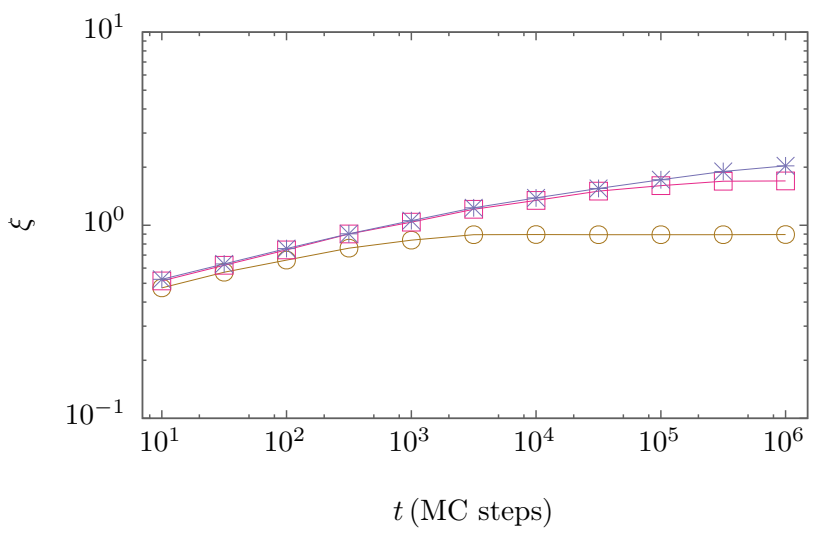

FIG. 8 . $\xi(t)$ for $T=1.0, T=1.2$ and $T=1.5$. Symbol codes are the same as in Fig. 3. Errors are smaller than symbol size.

of $t, \nu$ is strongly deceasing. The ratio $l^{3} / \xi^{3}$ is always greater than one, meaning that a jump is a pretty infrequent event in which a representative part of the spins in the box moves cooperatively.

Using an alternative definition of jumps, by changing the value of $\gamma$, also leads to the same qualitative behavior. For instance, a higher $\gamma$ (more restrictive definition, then fewer jumps) makes effective exponent of $\nu$ more negative. For example, we can compare the results for $\gamma=5$, shown in Fig. 9, with those for $\gamma=3$, in Fig. 7 . Notice that, in first case, $\nu$ decreases by two orders of magnitude, while it decreases about one order of magnitude for the second. On the other hand, we can observe that the relation $\nu r=1$ does not hold about $t=10^{5}$ The reason for this behavior is that, for $\gamma=5$ the average of the rest time $\langle r\rangle$ is of the order of $10^{5}$ (while $\langle r\rangle \simeq 10^{3}$ for $\gamma=3$ ). Now if we calculate the slope in the range $10^{6}<t<10^{7}$, we recover opposite slopes, see table II

We have also studied jump energy and jump magnetization, defined as the absolute value of the energy and magnetization differences of each box before and after the jump. These variables do not sensitively evolve with $t$. The energy and magnetization of every box also become stationary well before $\tau_{e}$. Varying $\gamma$, we found that less frequent jumps are related to greater changes in magnetization and energy.

We have explored the use of an alternative criteria of jump, with the same threshold for every box. That is, by considering that box $i$ is jumping at $t$ when $O_{i}(t)<B$, with the same constant $B$ for all boxes. We find that, since every box has a different quenched disorder, we get fast boxes jumping very frequently and slow boxes, jumping few times in the simulation time. So this definition gives some unwanted results; related to the fact that rest time is severely underestimated at long $t$.

Finally, we have tried other jump criteria by defining a jump when magnetization differences overcome a certain threshold. We have found similar results to the ones presented here using this alternative criteria.

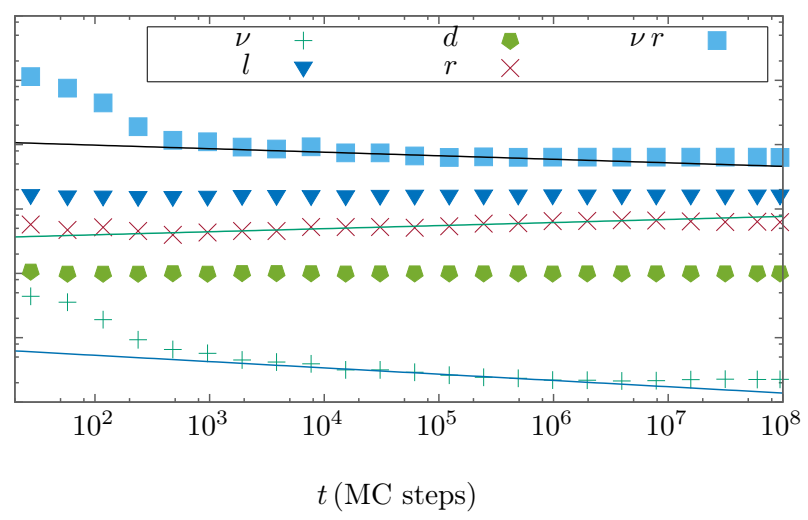

FIG. 9. (Color online) Time evolution of jump properties for for $\gamma=5$. Sets of data points are shifted vertically to facilitate comparison. Let us remark that $\langle r\rangle \simeq 10^{5}$. Errors are smaller than symbol size.

The results of these tests give support to the idea that, in spite of the manner we define a jump, we always get a jump frequency which depends strongly on time, but jump properties (with the exception of $r$, at long times) reach stationarity much before the equilibration time.

\section{DISCUSSION AND CONCLUSION}

In this work, we generalize the concept of jump, introduced in the context of glass formers [16], to the case of spin glasses. We divide the system into boxes, and define a jump as a cooperative spin flipping making the overlap function of a box to decay below a certain amount in a small time interval $\delta t$.

We study the statistical properties of these jumps as a function of the waiting time $t$ after a quench, for a 3D Edwards-Anderson model of spin glass.

When this system is quenched to a temperature $T_{F}$ higher than the glass transition temperature $T_{g} \simeq 0.95$, it reaches equilibrium after a characteristic time $\tau_{e}$, which we determine numerically from the stabilization of the global two-time correlation function $C(t+\Delta t, t)$. We confirm that every statistical property of jumps becomes independent of time for $t>\tau_{e}$. Interestingly however, in the dynamics of relaxation towards equilibrium, we find that most one-time microscopic observables related to jumps become nearly stationary well before $\tau_{e}$. The exception being jump frequency, which is a clearly de- 
creasing quantity for times much closer to the equilibration time, and rest time, which evolves inversely to jump frequency, and, in this sense, it is not an independent quantity. In this sense, our results are qualitatively more similar to those discussed in [22] than in [16]; the latter reporting a constant rest time for a kind of structural glass former. While the strongly decreasing jump frequency, with almost $t$ insensitive jump lengths and duration, as shown in [16], is a interesting result, it would be desirable to perform molecular-dynamics simulations for that system using longer simulation times, or smaller rest times (via less restrictive definition of jumps), and check whether an inverse relationship among $r$ and $\nu$ exists, after a transient of some $\langle r\rangle$ 's. For $T_{F}<T_{g}$, when the equilibration time diverges, we observe that all the measured microscopic observables depend on time, in the time interval that corresponds to our simulations. However, while jump duration and jump size change very slowly, jump frequency decreases much faster. This suggests that in the glass phase the number of jumps always decreases but jumps themselves are not $t$ sensitive at long times.

These conclusions do not depend qualitatively on the chosen values of $\delta t$, system size, box size nor the details of the criteria to define a jump. In particular, if jumps are defined as a function of magnetization or energy changes instead of overlap changes, similar results are found.

\section{ACKNOWLEDGMENTS}

Data on graphs were averaged using gs_gav program from glsim package [40]. This research was supported in part by the Consejo Nacional de Investigaciones Científicas y Técnicas (CONICET), and the Universidad Nacional de Mar del Plata. JLI is grateful for the financial support and hospitality of the Abdus Salam International Centre for Theoretical Physics (ICTP), where part of this article was written.

\section{APPENDIX: MACROSCOPIC RELAXATION TIME}

From $C(t+\Delta t, t)$ data, we need to estimate the value of $\tau_{a}(t)$, the value of $\Delta t$ for which $C=0.2$. Since data is subject to statistical error, it would be desirable to have an analytic expression to fit $C(t+\Delta t, t)$, such that, for a given $t$, all available information contributes to the fit. In [39], the shape of $C_{\infty}(\Delta t) \doteq \lim _{t \rightarrow \infty} C(t+\Delta t, t)$ was studied for the $\pm J$ Edwards-Anderson Model below $T_{g}$. Two alternative expressions where proposed: a power law, $C_{\infty}(\Delta t)=q_{E A}+A t^{\beta}$ and a logarithmic $C_{\infty}(\Delta t)=q_{E A}+\frac{A}{B+\log (t)}$, which performed similarly for their data. We have tried them setting $q_{E A}=0$. None of them seemed to work for our results $\mathrm{n}$ the whole range of times. So we have tried the simplest available approximation, $C=A+b \log (t)$. Clearly, the asymptotic form
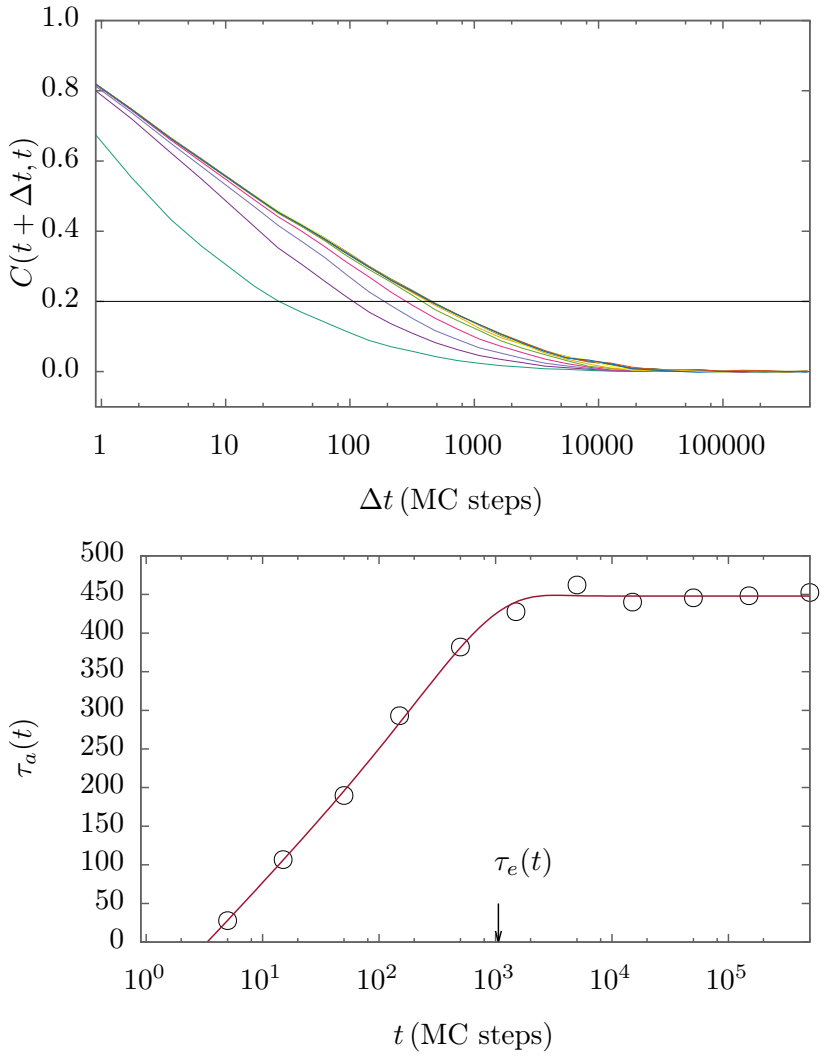

FIG. 10. (Color online) Top: Global two-time correlations for $T_{F}=1.5$, and several waiting times. From left to right, $t=5 ., 1.510,5.10,1.510^{2}, 5.10^{2}, 1.510^{3}, 5.10^{3}, 1.510^{4}$, $5.10^{4}, 1.510^{5}, 5.10^{5} \mathrm{MC}$ steps. The horizontal line corresponds to $C(t+\Delta t, t)=0.2$. Bottom: $\tau_{a}$ as a function of $t$ for $T_{F}=1.5$ (black circles). The (red) line corresponds to the fitting function $\left(C_{1}+C_{2} \log (t)\right) e^{-t / C_{3}}+C_{4}\left(1-e^{-t / C_{3}}\right)$. The arrow indicates $\tau_{e}=C_{3}$. Relative errors are of the same order of magnitude as those in Fig. 2-bottom.

of this function is nonphysical, so we use it as a mere approximation of our data on a limited range. We have restricted our data to points where $0.1<C<0.4$ for large $t$, and smaller ranges for shorter times. This approximation gave better results ( based on the comparison of goodness of fit divided by the degrees of freedom, $\chi^{2} / n$, where $n$ is the number of points used for the fit). We have also checked that $\tau_{e}(T)$ do not depend sensitively on the range of the value of $K$.

From $\tau_{a}(t)$, we have to extract $\tau_{e}(T)$. Looking at the form of the data, Fig. 2, we have proposed a simple way to describe it: $F(t)=\left(C_{1}+C_{2} \log (t)\right) e^{-t / C_{3}}+C_{4}\left(1-e^{-t / C_{3}}\right)$. For short times, $F(t)$ is described by the simplest growing function we could use, $C_{1}+C_{2} \log (t)$, at very long times, it goes to a constant $C_{4}$. The change of regime (from growing to constant) is given at $t=C_{3}$, so we estimate $\tau_{e}(T)=C_{3}$. This procedure also works for other temperatures, see Fig. 10, which shows the same information as Fig. 2, for $T=1.5$. 
We have also checked that $\tau_{e}(T)$ do not depend sensitively on the range of the value of $K$.

As mentioned in the text, $\tau_{e}(T)$ obtained this way is compatible with energy results, see Fig. 11.

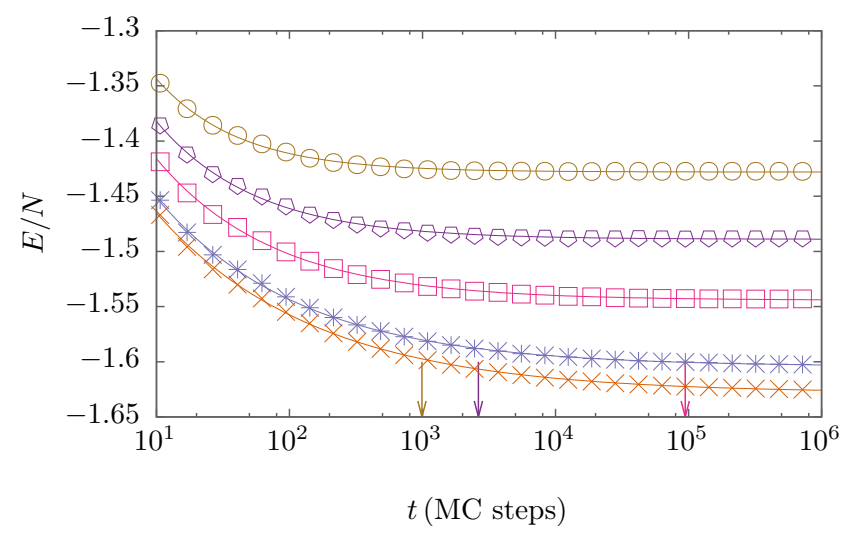

FIG. 11. Points: Energy as a function of time. Line: data fit using $e+A(T) t^{b}(T)$. Errors are smaller than symbol size.

[1] Ludovic Berthier and Giulio Biroli, "Theoretical perspective on the glass transition and amorphous materials," Rev. Mod. Phys. 83, 587-645 (2011).

[2] Tommaso Castellani and Andrea Cavagna, "Spin-glass theory for pedestrians," Journal of Statistical Mechanics: Theory and Experiment 2005, P05012 (2005).

[3] J A Mydosh, "Spin glasses: redux: an updated experimental/materials survey," Reports on Progress in Physics 78, 052501 (2015).

[4] M. Tarzia and M. A. Moore, "Glass phenomenology from the connection to spin glasses," Phys. Rev. E 75, 031502 (2007).

[5] Claudio Chamon, Leticia Cugliandolo, Gabriel Fabricius, José Luis Iguain, and Eric R. Weeks, "From particles to spins : Eulerian formulation of supercooled liquids and glasses," PNAS 105, 15263-15268 (2008).

[6] M. A. Moore and J. Yeo, "Thermodynamic glass transition in finite dimensions," Phys. Rev. Lett. 96, 095701 (2006).

[7] T. R. Kirkpatrick and P. G. Wolynes, "Stable and metastable states in mean-field potts and structural glasses," Phys. Rev. B 36, 8552-8564 (1987).

[8] J.P. Bouchaud and M. Mzard, "Self induced quenched disorder: a model for the glass transition," J. Phys. I France 4, 1109-1114 (1994).

[9] E Marinari, G Parisi, and F Ritort, "Replica field theory for deterministic models. ii. a non-random spin glass with glassy behaviour," Journal of Physics A: Mathematical and General 27, 7647 (1994).

[10] "Lectures notes in physics, complex behaviour of glassy systems, proceedings of the xiv sitges conference," in Lectures notes in Physics, Vol. 492, edited by Miguel Rubí and Conrado Pérez-Vicente (Springer, 1997).

[11] Kob, W. and Barrat, J.-L., "Fluctuations, response and aging dynamics in a simple glass-forming liquid out of equilibrium," Eur. Phys. J. B 13, 319-333 (2000).

[12] Giorgio Parisi, "Short-time aging in binary glasses," Journal of Physics A: Mathematical and General 30, L765 (1997).

[13] Enzio Andrejew and Jrg Baschnagel, "Aging effects in glassy polymers: a monte carlo study," Physica A: Statistical Mechanics and its Applications 233, 117 - 131 (1996).

[14] K. Vollmayr-Lee, J. A. Roman, and J. Horbach, "Aging to equilibrium dynamics of sio $_{2}$," Phys. Rev. E $\mathbf{8 1}$, 061203 (2010).

[15] Azita Parsaeian and Horacio E. Castillo, "Equilibrium and nonequilibrium fluctuations in a glass-forming liquid," Phys. Rev. Lett. 102, 055704 (2009).

[16] Katharina Vollmayr-Lee, Robin Bjorkquist, and Landon M. Chambers, "Microscopic picture of aging in $\operatorname{sio}_{2}$," Phys. Rev. Lett. 110, 017801 (2013).

[17] M. Warren and J. Rottler, "Atomistic mechanism of physical ageing in glassy materials," EPL (Europhysics Letters) 88, 58005 (2009).

[18] Mya Warren and Jörg Rottler, "Microscopic view of accelerated dynamics in deformed polymer glasses," Phys. Rev. Lett. 104, 205501 (2010).

[19] Helfferich, J., Vollmayr-Lee, K., Ziebert, F., Meyer, H., and Baschnagel, J., "Glass formers display universal non-equilibrium dynamics on the level of single-particle jumps," EPL 109, 36004 (2015).

[20] Anton Smessaert and Jörg Rottler, "Distribution of local relaxation events in an aging three-dimensional glass: Spatiotemporal correlation and dynamical heterogeneity," Phys. Rev. E 88, 022314 (2013).

[21] P Sibani and H. Jeldtoft Jensen, "Intermittency, aging and extremal fluctuations," Europhysics Letters (EPL) 69, 563-569 (2005), arXiv:0403212 [cond-mat].

[22] Nima H Siboni, Dierk Raabe, and Fathollah Varnik, 
"Aging in amorphous solids : A study of the first passage time and persistence time distributions," 2, 1-6, arXiv:arXiv:1407.5271v2.

[23] Raffaele Pastore, Antonio Coniglio, and Massimo Pica Ciamarra, "From cage-jump motion to macroscopic diffusion in supercooled liquids," Soft Matter 10, 57245728 (2014).

[24] R Pastore, A Coniglio, A de Candia, A Fierro, and M Pica Ciamarra, "Cage-jump motion reveals universal dynamics and non-universal structural features in glass forming liquids," Journal of Statistical Mechanics: Theory and Experiment 2016, 054050 (2016).

[25] Massimo Pica Ciamarra, Raffaele Pastore, and Antonio Coniglio, "Particle jumps in structural glasses," Soft Matter 12, 358-366 (2016).

[26] S F Edwards and P W Anderson, "Theory of spin glasses," Journal of Physics F: Metal Physics 5, 965 (1975).

[27] W.Y Ching and D.L Huber, "Monte carlo studies of the internal energy and specific heat of a classical heisenberg spin glass," Physics Letters A 59, 383 - 384 (1976).

[28] K. Binder and D. Stauffer, "Monte carlo simulation of a three-dimensional spin glass," Physics Letters A 57, 177 - 179 (1976).

[29] Ludovic D C Jaubert, Claudio Chamon, Leticia F Cugliandolo, and Marco Picco, "Growing dynamical length, scaling, and heterogeneities in the 3d edwardsanderson model," Journal of Statistical Mechanics: Theory and Experiment 2007, P05001 (2007).

[30] Markus Manssen, Alexander K. Hartmann, and A. P. Young, "Nonequilibrium evolution of window overlaps in spin glasses," Phys. Rev. B 91, 104430 (2015).

[31] F. Romá and S. Risau-Gusman, "Backbone structure of the edwards-anderson spin-glass model," Phys. Rev. E 88, 042105 (2013).

[32] Matteo Lulli, Giorgio Parisi, and Andrea Pelissetto, "Out-of-equilibrium finite-size method for critical behavior analyses," Phys. Rev. E 93, 032126 (2016).

[33] Wenlong Wang, Jonathan Machta, and Helmut G. Katzgraber, "Evidence against a mean-field description of short-range spin glasses revealed through thermal boundary conditions," Phys. Rev. B 90, 184412 (2014).

[34] Luis Antonio Fernández and Víctor Martín-Mayor, "Testing statics-dynamics equivalence at the spin-glass transition in three dimensions," Phys. Rev. B 91, 174202
$(2015)$

[35] F. Belletti, M. Cotallo, A. Cruz, L. A. Fernandez, A. Gordillo-Guerrero, M. Guidetti, A. Maiorano, F. Mantovani, E. Marinari, V. Martin-Mayor, A. Muoz-Sudupe, D. Navarro, G. Parisi, S. Perez-Gaviro, M. Rossi, J. J. Ruiz-Lorenzo, S. F. Schifano, D. Sciretti, A. Tarancon, R. Tripiccione, J. L. Velasco, D. Yllanes, and G. Zanier, "Janus: An fpga-based system for high-performance scientific computing," Computing in Science Engineering 11, 48-58 (2009).

[36] M. Baity-Jesi, R.A. Baos, A. Cruz, L.A. Fernandez, J.M. Gil-Narvion, A. Gordillo-Guerrero, D. Iiguez, A. Maiorano, F. Mantovani, E. Marinari, V. Martin-Mayor, J. Monforte-Garcia, A. Muoz Sudupe, D. Navarro, G. Parisi, S. Perez-Gaviro, M. Pivanti, F. RicciTersenghi, J.J. Ruiz-Lorenzo, S.F. Schifano, B. Seoane, A. Tarancon, R. Tripiccione, and D. Yllanes, "Janus ii: A new generation application-driven computer for spinsystem simulations," Computer Physics Communications 185, 550 - 559 (2014).

[37] F. Belletti, M. Cotallo, A. Cruz, L. A. Fernandez, A. Gordillo-Guerrero, M. Guidetti, A. Maiorano, F. Mantovani, E. Marinari, V. Martin-Mayor, A. Muñoz Sudupe, D. Navarro, G. Parisi, S. Perez-Gaviro, J. J. RuizLorenzo, S. F. Schifano, D. Sciretti, A. Tarancon, R. Tripiccione, J. L. Velasco, and D. Yllanes, "Nonequilibrium spin-glass dynamics from picoseconds to a tenth of a second," Phys. Rev. Lett. 101, 157201 (2008).

[38] Helmut G. Katzgraber, Mathias Körner, and A. P. Young, "Universality in three-dimensional ising spin glasses: A monte carlo study," Phys. Rev. B 73, 224432 (2006).

[39] F. Belletti, A. Cruz, L. A. Fernandez, A. GordilloGuerrero, M. Guidetti, A. Maiorano, F. Mantovani, E. Marinari, V. Martin-Mayor, J. Monforte, A. Muoz Sudupe, D. Navarro, G. Parisi, S. Perez-Gaviro, J. J. Ruiz-Lorenzo, S. F. Schifano, D. Sciretti, A. Tarancon, R. Tripiccione, and D. Yllanes, "An in-depth view of the microscopic dynamics of ising spin glasses at fixed temperature," Journal of Statistical Physics 135, 11211158 (2009).

[40] Tomás S. Grigera, "glsim: A general library for numerical simulation," Computer Physics Communications 182, $2122-2131$ (2011). 